\title{
Magnetic resonance imaging of the entorhinal cortex and hippocampus in mild cognitive impairment and Alzheimer's disease
}

A T Du, N Schuff, D Amend, M P Laakso, Y Y Hsu, W J Jagust, K Yaffe, J H Kramer, B Reed, D Norman, H C Chui, M W Weiner

Magnetic Resonance Unit, Department of Veterans Affairs Medical Center (114M), University of California, San Francisco 4150, Clement Street, San Francisco, CA 94121, USA

A T Du

N Schuff

D Amend

$\mathrm{M}$ W Weiner

Department of

Radiology,

N Schuff

D Norman

$\mathrm{M}$ W Weiner

Department of

Psychiatry

$\mathrm{M}$ W Weiner

K Yaffe

J H Kramer

Department of

Neurology

$\mathrm{MW}$ Weiner

K Yaffe

Department of

Medicine

$\mathrm{M}$ W Weiner

Departments of

Neurology and Clinical

Radiology, Kuopio

University Hospital,

Kuopio, Finland

M P Laakso

Department of

Radiology, Chang

Gung Memorial

Hospital, Taiwan

Y Y Hsu

Department of

Neurology, University

of California, Davis,

USA

W J Jagust

B Reed

Department of

Neurology, University of Southern

California, USA

H C Chui

Correspondence to: Dr M W Weiner

mweiner@itsa.ucsf.edu

Received 21 August 2000 and in revised form

26 March 2001

Accepted 18 April 2001

\begin{abstract}
Objectives-To explore volume changes of the entorhinal cortex (ERC) and hippocampus in mild cognitive impairment (MCI) and Alzheimer's disease (AD) compared with normal cognition (NC); to determine the powers of the ERC and the hippocampus for discrimination between these groups.
\end{abstract}

Methods-This study included 40 subjects with NC, 36 patients with MCI, and 29 patients with AD. Volumes of the ERC and hippocampus were manually measured based on coronal T1 weighted MR images. Global cerebral changes were assessed using semiautomatic image segmentation. Results-Both ERC and hippocampal volumes were reduced in MCI (ERC 13\%, hippocampus $11 \%, \mathrm{p}<0.05)$ and AD (ERC $39 \%$, hippocampus $27 \%$, p<0.01) compared with NC. Furthermore, AD showed greater volume losses in the ERC than in the hippocampus $(p<0.01)$. In addition, $A D$ and MCI also had cortical grey matter loss $(p<0.01)$ and ventricular enlargement $(p<0.01)$ when compared with NC. There was a significant correlation between ERC and hippocampal volumes in MCI and AD (both p<0.001), but not in NC. Using ERC and hippocampus together improved discrimination between $\mathrm{AD}$ and $\mathrm{CN}$ but did not improve discrimination between MCI and NC. The ERC was better than the hippocampus for distinguishing MCI from AD. In addition, loss of cortical grey matter significantly contributed to the hippocampus for discriminating MCI and AD from NC.

Conclusions-Volume reductions in the ERC and hippocampus may be early signs of AD pathology that can be measured using MRI.

(F Neurol Neurosurg Psychiatry 2001;71:441-447) tive impairment; Alzheimer's disease

Pathological studies of brains from patients with Alzheimer's disease (AD) showed the greatest neurodegenerative changes in the entorhinal cortex (ERC) and hippocampus compared with other brain regions. ${ }^{12}$ In addition, there is evidence that early $\mathrm{AD}$ pathology may start in the ERC, then progress to the hippocampus. ${ }^{3}$ In accordance with these findings, MRI studies have shown significant
Keywords: entorhinal cortex; hippocampus; mild cogni- atrophy of the ERC and hippocampus in patients with $\mathrm{AD},{ }^{4-14}$ in addition to generalised brain atrophy, loss of grey matter, and increased white matter lesions. ${ }^{15-17}$ In an attempt to determine which brain region showed the greatest change in $\mathrm{AD}$, several MRI studies compared changes in the ERC and hippocampus. ${ }^{4218}$ Using a small sample of subjects, Bobinski et al reported that changes in the ERC showed greater discrimination between patients with early $\mathrm{AD}$ and controls with normal cognition (NC) than changes in the hippocampus. ${ }^{18}$ But Frisoni et al reported that hippocampal changes had superior diagnostic accuracy over ERC changes in patients with $\mathrm{AD}$ compared with those with NC. ${ }^{12}$ By contrast, Juottonen et al and Xu et al found little difference between the ability of the ERC and the hippocampus to distinguish patients with $\mathrm{AD}$ from subjects with NC. ${ }^{419}$

Recently there has been increased interest in identifying patients at the earliest stages of $\mathrm{AD}$, so that effective treatment (when this is developed) can be initiated at an early stage. Therefore, neuroimaging studies have been performed on non-demented subjects who are at increased risk for $\mathrm{AD}$, including family history of $\mathrm{AD},{ }^{20}$ the APOE genotype, ${ }^{21}$ and nondemented patients with mild cognitive impairment (MCI). ${ }^{22-25}$ The hippocampus was found to be significantly reduced in subjects with MCI compared with those with NC. ${ }^{22} 23$ Recently, two publications addressed ERC volume in patients with MCI. Both reported that the ERC was significantly reduced in patients with MCI compared with those with NC and $\mathrm{Xu}$ et al found that the ERC and the hippocampus had roughly equivalent discrimination power between $\mathrm{MCI}^{1926}$ and $\mathrm{NC}$; however, the discrimination powers of ERC shown in these two papers were quite different. ${ }^{1926}$

Therefore, the overall goal of this study was to determine if changes in the ERC provided diagnostic information in addition to that obtained from the hippocampus and other brain structures in $\mathrm{AD}$ and MCI. Brain MRI measurements of the following volumes were quantified: ERC, hippocampus, cortical grey matter (cGM), subcortical GM (sGM), white matter (WM), sulcal CSF (sCSF), ventricular CSF (vCSF), and white matter signal hyperintensities (WMSH). Our specific goals were to determine: (1) Whether the ERC and hippocampal volumes are significantly reduced in patients with MCI compared with subjects 
with NC; (2) Whether ERC and hippocampal volumes are significantly reduced in patients with AD compared with patients with MCI; (3) Whether ERC volume is more sensitive than hippocampal volume to distinguish $\mathrm{AD}$ from MCI, AD from NC, and MCI from NC; (4) What is the difference in tissue segmentation between NC, MCI, and AD; (5) the extent to which ERC and hippocampal volumes are correlated with each other and the extent to which brain volume measurements are correlated with the cognitive impairment, measured by the mini mental state examination (MMSE). Finally, we compared the powers of the ERC and hippocampus to classify between groups and, in addition, assessed the value of using the ERC, hippocampus, and segmentation together for classification.

\section{Materials and methods}

SUBJECTS

There were 40 healthy elderly people with normal cognition (NC, 20 men, 20 women, age 75.1 (SD 4.3) years, MMSE 29.0 (SD 0.9)), 36 patients with mild cognitive impairment (MCI, 17 men, 19 women, age 75.1 (SD 8.2) years, MMSE 25.8 (SD 3.6)), and 29 patients with Alzheimer's disease (AD, 13 men, 16 women, age 75.8 (SD 5.1) years, MMSE 17.7 (SD 5.7)). The ERC and hippocampal volumes were manually measured in all subjects. Segmentation of brain tissues into cGM, sGM, WM, sCSF, vCSF, and WMSH was carried out in 39 subjects with NC (20 men, 19 women, age 75.3 (SD 4.2) years, MMSE 28.2 (SD $0.9), 27$ patients with MCI (14 men, 13 women, age 76.1 (SD 7.6) years, MMSE 26.2 (SD 3.5)), and 27 patients with $\mathrm{AD}$ (13 men, 14 women, age 75.5 (SD 5.1) years, MMSE 16.9 (SD 5.8)). All patients with NC had neurological and neuropsychological tests performed by the same staff at the recruitment centres that examined patients with MCI and those with $\mathrm{AD}$ and had test scores within the normal range. Furthermore, subjects with NC were included only if they had no clinical histories of alcoholism, psychiatric illnesses, epilepsy, hypertension, diabetes, major heart disease, or head trauma, and no sign on the MRI data of other major neurodegenerative diseases.

DIAGNOSIS OF MCI

The diagnostic team, based on comprehensive dementia evaluation that included history from the patients and a caregiver, neurological and mental status examinations of the patient, and neuropsychological testing determined that the patient's cognitive function was not normal, but that they did not meet DSM IV criteria for dementia. Examples of this situation would include a single, isolated cognitive deficit or a set of mild cognitive deficits insufficient to cause significant functional impairment. Neuropsychological testing was classified as normal or not according to the clinical judgment of neuropsychologists expert in the evaluation of dementia, and took into account all relevant factors including estimated premorbid functions. However, the final diagnosis was not solely based on the test interpretation. It was the consensus diagnosis of the clinicians based on all available evidence. Some of these patients did not meet the criteria for MCI published by Petersen et al. ${ }^{27}$

\section{DIAGNOSIS OF AD}

Alzheimer's disease was diagnosed according to the National Institute of Neurological and Communicative Disorders and StrokeAlzheimer's Disease and Related disorders Association (NINCDS/ADRDA) criteria, including probable and possible AD.

All patients with MCI and those with $\mathrm{AD}$ were recruited from the University of California, San Francisco and the University of California, Davis Alzheimer centre. Control subjects were recruited by posting flyers in the community. The cognitive function of each patient was screened with the MMSE. All patients were scanned using the same protocol. All patients or their guardians gave written informed consent before participating in the study, which was approved by the committees of human research at the University of California, San Francisco and the University of California, Davis.

MRI EXAMINATION

All studies were performed on a $1.5 \mathrm{~T}$ Magnetom VISION system (Siemens Inc, Iselin, NJ, USA) equipped with a standard quadrature head coil. The MRI protocol consisted of sagittal $\mathrm{T} 1$ weighted scout view images, oblique axial double spin echo (DSE) scans parallel to the axis of optic nerve, and volumetric magnetisation prepared rapid acquisition gradient echo (MP-RAGE) perpendicular to the DSE images yielding T1 weighted coronal images roughly perpendicular to the long axis of the hippocampus. The measurement parameters of DSE were TR/TE1/TE2 2500/20/80 $\mathrm{ms}$, field of view (FOV) $192 \times 256 \mathrm{~mm}^{2}$, matrix size $154 \times 256$, in plane resolution $1.25 \times 1.00$ $\mathrm{mm}^{2}$, and $3 \mathrm{~mm}$ slice thickness covering the whole brain from the vertex to the most inferior part of the cerebellum. The measurement parameters of MP-RAGE were TR/TI/TE $10 / 250 / 4$ ms with a 15 degree flip angle, FOV $192 \times 256 \mathrm{~mm}^{2}$, matrix size $192 \times 256$, and 1.4 $\mathrm{mm}$ thick partition, yielding $1.0 \times 1.0 \times 1.4 \mathrm{~mm}^{3}$ spatial resolution.

MEASUREMENT OF ERC AND HIPPOCAMPAL VOLUME

Quantitative volumes of the ERC and hippocampus were obtained by manually drawing the boundary of the structures as seen in the coronal T1 weighted MP-RAGE images shown in figure 1. Measurement of ERC volume was performed according to the protocol developed by Insausti et $a .^{28}$ Briefly, ERC was measured from one section caudal to the level of the limen insulae, and until the section behind the posterior limit of the gyrus intralimbicus. The medial margin of the ERC was marked along the ventral border of the gyrus semilunaristhat is, the fundus of the sulcus semiannularis. The lateral margin of the ERC was in the medial bank of the collateral sulcus, where it 


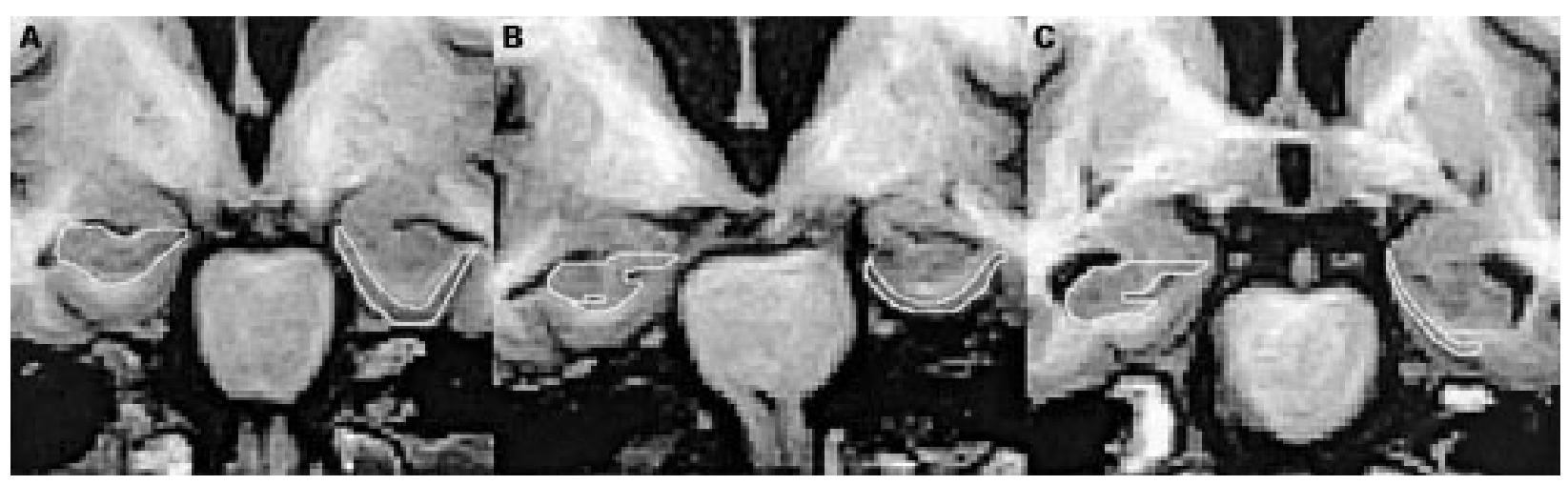

Figure 1 Entorhinal cortex (ERC) (right) and hippocampal (left) volume measurement in MP-RAGE images. (A) Normal cognition (NC); (B) mild cognitive impairment (MCI); (C) Alzheimer's disease (AD).

borders the perirhinal cortex. The borders of the ERC and perirhinal cortex depended on the depth of the collateral sulcus. Boundaries of the hippocampus were drawn following the guidelines of Watson et $a l,{ }^{29}$ including the hippocampus proper, dentate gyrus, subiculum, fimbria, and alveus. One rater (ATD), who was blinded to the diagnosis and all other clinical information, performed all measurements of the ERC and hippocampus. Rater reliability was determined by marking the ERC and hippocampus of 10 subjects twice and expressing the coefficients of variation $(\mathrm{CoV})$. The CoV was $2.6 \%$ for the ERC and $1.0 \%$ for the hippocampus.

BRAIN TISSUE SEGMENTATION

Before tissue segmentation was performed, MRI data were corrected for B1 inhomogeneity with a low frequency intensity filter, as described by Tanabe et al. ${ }^{15}$ Complications with the potential problem of cross talking between slices of DSE images was avoided by acquiring DSE using an interleaved slice selection mode and a $2500 \mathrm{~ms}$ delay time between interleaved acquisitions. Slice cross talking is not a problem for MP-RAGE, which is performed in a volumetric fashion without selection of slices. After the scalp was manually stripped, both images of the double spin echo and the 3D T1 weighted MP-RAGE were coregistered. Then a semiautomatic program combining threshold methods and discrimination analysis incorporating the data from each pixel from both images of the T2 weighted MRI and the 3D T1 weighted MRI was used to segment brain tissues. ${ }^{15}$ Total intracranial volume (TIV) was measured from the top of the brain to the slice where the cerebral peduncles appeared. Brain tissues were automatically segmented to grey matter (GM), white matter (WM), and CSF. Subsequently, an operator manually separated GM to cGM and sGM, and CSF to sCSF and vCSF. Finally, WMSH was manually separated from WM.

NORMALISATION

To account for variation in TIV, the volumes of the ERC, hippocampus, and segmentation data of each subject were normalised to individual TIV (TIV $)$, according to the formula:

$$
\mathrm{VOL}^{\mathrm{i}, \mathrm{n}}=\mathrm{VOL}^{\mathrm{i}} \times \mathrm{TIV}^{\mathrm{m}} / \mathrm{TIV}^{\mathrm{i}}
$$

where $\operatorname{VOL}^{\mathrm{i}, \mathrm{n}}$ is the normalised volume of a subject, $\mathrm{VOL}^{\mathrm{i}, \mathrm{n}}$ is the non-normalised volume, and TIV $^{\mathrm{m}}$ is the mean TIV of all subjects.

\section{STATISTICS}

Statistical analysis was guided by a statistician and was performed using S-Plus software (Mathsoft Inc, Seattle, USA). Group effects of ERC and hippocampus adjusted by age and sex were tested using analysis of variance (ANOVA). Percent volume loss of the ERC and hippocampus within MCI and AD groups were compared using paired $t$ test. Our a priori hypothesis of differences between the groups in ERC and hippocampal volumes was tested using $\alpha=0.05$ as level of significance. By contrast, other hypotheses about differences between groups in segmentation measures (which were not a priori) were tested using an adjusted $\alpha$ level of $0.05 / 6 \approx 0.01$ to account for the six comparisons using segmentation data. Pearson correlation coefficients were used to analyze the correlation between volumes of the ERC, hippocampus, segmented structures, and MMSE in each group. Only subjects who had complete ERC and hippocampal volumetry and tissue segmentation were included for classification analysis. The powers of the ERC and hippocampus for group classification were tested using logistic regression analysis, followed by McNemar's $\chi^{2}$ statistics to verify whether the differences were significant, and receiver operator characteristics (ROC) analysis. Stepwise logistic regression analysis was used to test combinations of the ERC, hippocampus, and segmentation measures for the power to discriminate between the groups.

\section{Results}

DEMOGRAPHICS

Table 1 shows that all groups were well matched according to age $(F=0.14, \mathrm{p}=0.87)$ and sex $\left(\chi^{2}=0.18, p=0.91\right)$. The MMSE score of patients with AD (17.7 (SD 5.7) was significantly lower than that of patients with MCI (25.8 (SD 3.6) $(\mathrm{p}<0.001)$ and those with NC (29.0 (SD 0.9) $(\mathrm{p}<0.001)$, and the MMSE score of patients with MCI was significantly lower than that of patients with NC $(p<0.001)$.

ERC AND HIPPOCAMPAL VOLUMES

Figure 2 shows the percentage changes of total ERC and hippocampal volumes for all subjects relative to mean volumes of NC, demonstrating 
Table 1 Demographics

\begin{tabular}{llllll}
\hline Category & No of patients & Male & Female & Age & MMSE \\
\hline NC & 40 & 20 & 20 & $75.1(4.3)$ & $29.0(0.9)$ \\
MCI & 36 & 17 & 19 & $75.1(8.2)$ & $25.8(3.6)^{\star}$ \\
AD & 29 & 13 & 16 & $75.8(5.1)$ & $17.7(5.7)^{\star} \dagger$ \\
\hline
\end{tabular}

Values in parentheses are SD

${ }^{\star} \mathrm{p}<0.001$ for $\mathrm{MCI} v \mathrm{NC}$, and $\mathrm{AD} v \mathrm{NC}$

$\mathrm{tp}<0.001$ for $\mathrm{MCI} v \mathrm{AD}$.

$\mathrm{NC}=$ Normal cognition; $\mathrm{MCI}=$ mild cognitive impairment; $\mathrm{AD}=\mathrm{Alzheimer}$ 's disease; MMSE $=$ mini mental state examination.

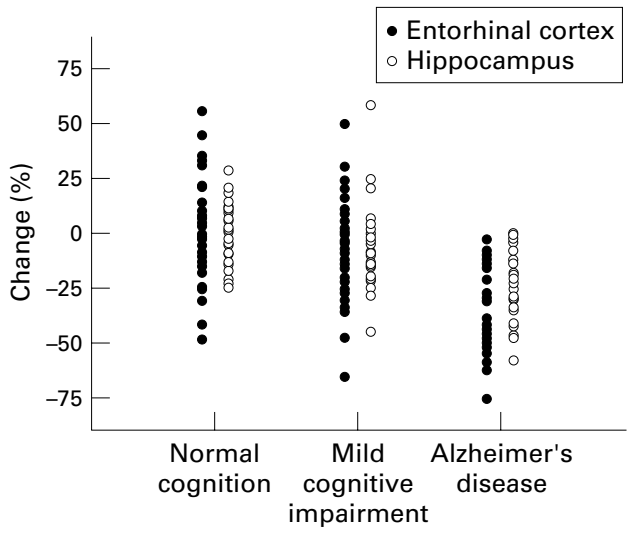

Figure 2 Percentage changes from the normal mean volumes of entorhinal cortex and hippocampus in normal cognition, mild cognitive impairment, and Alzheimer's disease. This figure shows that the most severe volume loss is the entorhinal cortex in Alzheimer's disease.

the prominent ERC volume losses in AD. Table 2 shows volumes of the ERC and hippocampus in NC, MCI, and AD groups. Total ERC volume of patients with NC was 2726 (SD 608) $\mathrm{mm}^{3}$. This was significantly reduced by $13 \%$ to 2385 (SD 632) $\mathrm{mm}^{3}$ in patients with MCI $(F=4.6, \mathrm{p}<0.05)$ and by $39 \%$ to 1662 (SD 501) $\mathrm{mm}^{3}$ in patients with $\mathrm{AD}(F=58.9$, $\mathrm{p}<0.01)$. When $\mathrm{AD}$ was compared with $\mathrm{MCI}$, the ERC was $30 \%$ significantly smaller in AD $(F=29.1, \mathrm{p}<0.01)$. Total hippocampal volume of patients with NC was 6327 (SD 799) $\mathrm{mm}^{3}$. This was significantly reduced by $11 \%$ to 5657 (SD 864) $\mathrm{mm}^{3}$ in patients with MCI $(F=6.8$, $\mathrm{p}<0.05$ ) and $27 \%$ to 4595 (SD 1009) $\mathrm{mm}^{3}$ in those with $\mathrm{AD}(F=63.2, \mathrm{p}<0.01)$. When $\mathrm{AD}$ was compared with MCI, hippocampus was $19 \%$ significantly smaller in $\mathrm{AD} \quad(F=18.9$, $\mathrm{p}<0.01)$. Furthermore, when $\mathrm{AD}$ was compared with NC, $39 \%$ reduction of the ERC was significantly greater than $27 \%$ reduction of hippocampus (paired $t$ test, $\mathrm{p}=0.01$ ). However,

Table 2 Volumes (mean (SD)) of entorhinal cortex and hippocampus

\begin{tabular}{|c|c|c|c|c|c|}
\hline & \multirow{2}{*}{$\begin{array}{l}\text { NC }(n=40) \\
\text { Volume }\left(\mathrm{mm}^{3}\right)\end{array}$} & \multicolumn{2}{|l|}{$M C I(n=36)$} & \multicolumn{2}{|l|}{$A D(n=29)$} \\
\hline & & Volume $\left(\mathrm{mm}^{3}\right)$ & $\%$ Change & Volume $\left(\mathrm{mm}^{3}\right)$ & $\%$ Change \\
\hline \multicolumn{6}{|l|}{ ERC: } \\
\hline Left & $1342(363)$ & $1153(347)^{\star \star}$ & 14 & $795(286)^{\star} \dagger$ & 41 \\
\hline Right & $1383(310)$ & $1231(335)^{\star}$ & 11 & $867(262)^{\star} \dagger$ & 37 \\
\hline Total & $2726(608)$ & $2385(632)^{\star \star}$ & 13 & $1662(501)^{\star} \dagger$ & 39 \\
\hline \multicolumn{6}{|l|}{ HP: } \\
\hline Left & $3135(403)$ & $2783(511)^{\star}$ & 11 & $2231(506)^{\star} \dagger$ & $29 \neq$ \\
\hline Right & $3191(432)$ & $2874(401)^{\star}$ & 10 & $2364(545)^{\star} \dagger$ & $26 \ddagger$ \\
\hline Total & $6327(799)$ & $5657(864)^{\star}$ & 11 & $4595(1009)^{\star} \dagger$ & $27 \ddagger$ \\
\hline
\end{tabular}

$\%$ Change is compared with NC.

${ }^{\star} \mathrm{p}<0.01 ;{ }^{\star \star} \mathrm{p}<0.05$ for MCI $v \mathrm{NC}$, and $\mathrm{AD} v \mathrm{NC}$.

$\mathrm{tp}<0.01$ for MCI $v \mathrm{AD}$.

$\ddagger \mathrm{p}<0.01$ for $\%$ change of ERC larger than hippocampus in each group.

$\mathrm{NC}=$ Normal cognition; $\mathrm{MCI}=$ mild cognitive impairment; $\mathrm{AD}=$ Alzheimer's disease $\mathrm{ERC}=$ entorhinal cortex; HP=hippocampus; Left=left side; Right=right side. when MCI was compared with NC, $13 \%$ reduction of the ERC was not significantly different from $11 \%$ hippocampal reduction in MCI (paired $t$ test, $\mathrm{p}>0.05$ ). In addition, when patients with $\mathrm{AD}$ were compared with those with MCI, 30\% reduction of the ERC was significantly greater than the $19 \%$ reduction of the hippocampus (paired $t$ test, $\mathrm{p}<0.05$ ). There was no significant group by side interaction neither for ERC volume changes $(F=0.07$, $\mathrm{p}=0.93$, ANOVA) nor for hippocampal volume changes $(F=0.12, \mathrm{p}=0.89$, ANOVA), providing no evidence for a laterality effect in ERC and hippocampal atrophy.

BRAIN SEGMENTATION

Table 3 shows that patients with MCI had significant cGM losses $(F=10.8, \mathrm{p}<0.01)$, vCSF enlargement $(F=5.3, \mathrm{p}<0.01)$, and WMSH increases $(F=7.0, \mathrm{p}<0.01)$ when compared with subjects with NC. As expected, compared with the NC group, the AD group had significant cGM loss $(F=33.7, \mathrm{p}<0.01)$, WM reduction $(F=16.2, \mathrm{p}<0.01)$, sCSF enlargement $(F=25.6, \mathrm{p}<0.01)$, and vCSF enlargement $(F=21.2, \mathrm{p}<0.01)$. In addition, when the $\mathrm{AD}$ group was compared with the MCI group, there were cGM and WM losses, and sCSF and vCSF increases; however, only WM reduction was significant $(F=9.7, \mathrm{p}<0.01)$.

CORRELATION

Figure 3 shows the correlation of ERC and hippocampal volumes in MCI and AD. There was significant correlation between the ERC and hippocampus in MCI $(r=0.66, \mathrm{p}<0.001)$ and $\mathrm{AD}(r=0.68, \mathrm{p}<0.001)$. However, there was no significant correlation between the ERC and hippocampus in NC $(r=0.25$, $\mathrm{p}=0.28$ ).

When all subjects were combined, there was a significant correlation between MMSE and the ERC $(r=0.48, \mathrm{p}<0.001)$, and MMSE and hippocampus $(r=0.48, \mathrm{p}<0.001)$, as shown in figure 4 . However, there was no significant correlation between MMSE and the ERC, and MMSE and hippocampus in any of the individual groups. When correlation was performed among the ERC, hippocampus, and segmentation data, only the hippocampus and cGM $(p<0.05)$ in NC and the ERC and cGM in MCI $(p<0.05)$ were correlated.

Table 3 Segmentation data

\begin{tabular}{lccc}
\hline & $N C(n=39)$ & $M C I(n=27)$ & $A D(n=27)$ \\
\hline cGM & $509(27)$ & $478(49)^{\star \star}$ & $462(39)^{\star \star}$ \\
sGM & $18(4)$ & $17(4)$ & $17(4)$ \\
WM & $462(30)$ & $458(27) \dagger$ & $431(35)^{\star \star}+$ \\
sCSF & $266(44)$ & $286(49)$ & $321(49)^{\star \star}$ \\
vCSF & $48(14)$ & $59(23)^{\star \star}$ & $66(18)^{\star \star}$ \\
WMSH & $6(6)$ & $14(17)^{\star \star}$ & $12(16)$ \\
\hline
\end{tabular}

$\star \star \mathrm{p}<0.01$ for NC with MCI or AD

$t \mathrm{p}<0.01$ for MCI with AD.

Values are mean (SD $\left.\left(\mathrm{cm}^{3}\right)\right)$. NC=Normal cognition; $\mathrm{MCI}=$ mild cognitive impairment; $\mathrm{AD}=$ Alzheimer's disease; cGM=cortical grey matter; sGM=subcortical grey matter; $\mathrm{WM}=$ white matter; $\mathrm{sCSF}=$ sulcal $\mathrm{CSF} ; \mathrm{vCSF}=$ ventricular $\mathrm{CSF}$; $\mathrm{WMSH}=$ white matter signal hyperintensities. 


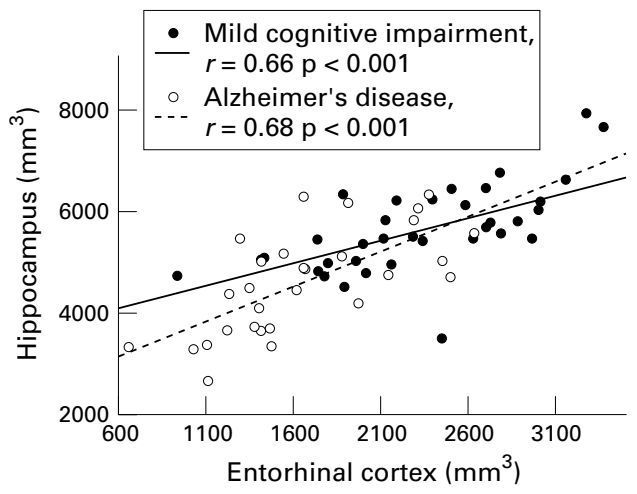

Figure 3 Correlation between entorhinal cortex and hippocampal volumes in mild cognitive impairment and Alzheimer's disease.

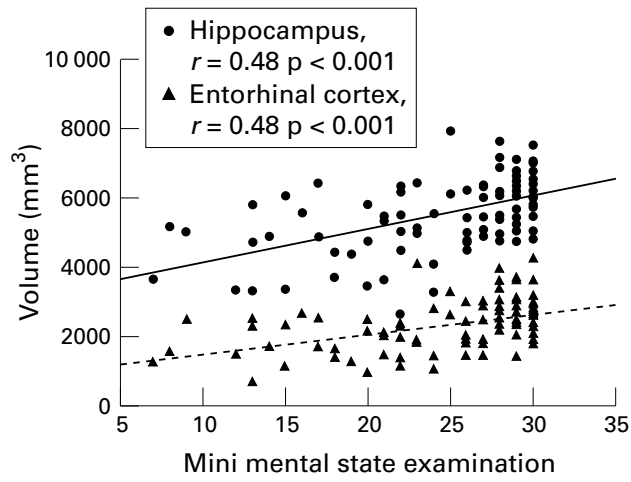

Figure 4 Correlation of mini mental state examination with the entorhinal cortex and with the hippocampus in the whole group.

CLASSIFICATION, SENSITIVITY, AND SPECIFICITY ANALYSIS

To compare the ERC and hippocampal volumes for their power to distinguish NC, $\mathrm{MCI}$, and $\mathrm{AD}$, logistic regression analysis was used to predict group memberships either with the ERC or hippocampus as independent variables. The overall classification between MCI and NC was $66 \%$ with the ERC and $70 \%$ with the hippocampus. The classification with the hippocampus was significantly better than with the ERC ( $<<0.05$, McNemar). The overall classification between $\mathrm{AD}$ and $\mathrm{NC}$ was $83 \%$ with the ERC and $86 \%$ with hippocampus. In this case, however, the difference between the ERC and hippocampus was not significant ( $p>0.05$, McNemar), implying that the discriminatory powers of the ERC and hippocampus were comparable. Finally, the overall classification between $\mathrm{AD}$ and $\mathrm{MCI}$ was $74 \%$ with the ERC and $72 \%$ with hippocampus and classification with the ERC was better than with hippocampus ( $p<0.05, \mathrm{McNemar})$.
To compare sensitivity and specificity between the ERC and hippocampus for group classification, ROC analysis was performed. Discriminating between MCI and NC, the area under the curve (AUC) of the ROC was 0.64 with the ERC and 0.71 with the hippocampus. Discriminating between $\mathrm{AD}$ and NC, AUC was 0.92 with the ERC and 0.91 with the hippocampus. Finally, discriminating between $\mathrm{AD}$ and MCI, AUC was 0.83 with the ERC and 0.79 with the hippocampus.

Finally, we employed stepwise logistic regression analysis to explore combinations of the ERC, hippocampus, and segmentation measurements for their powers to discriminate between the groups. Firstly, the hippocampus was added to the regression model, then the ERC was added to test whether the ERC significantly contributed to the discrimination, finally, segmented structures were added to test which segmented structure significantly contributed to the discrimination. The results are listed in table 4. Mild cognitive impairment and NC were classified with $52 \%$ sensitivity, $79 \%$ specificity, and an overall classification of $70 \%$ using the hippocampus $(\mathrm{p}<0.01)$ alone in the stepwise logistic regression model. Adding the ERC to the model did not improve classification $(p=0.31)$. Finally, when segmentation data were added to the model, cGM $(p<0.05)$ significantly contributed to the discrimination and increased sensitivity to $59 \%$, specificity to $82 \%$, and overall classification to $77 \%$. AD and NC were classified with $78 \%$ sensitivity, $90 \%$ specificity, and an overall classification of $86 \%$ using the hippocampus $(\mathrm{p}<0.01)$ alone. Adding the ERC $(p<0.01)$ significantly increased sensitivity to $78 \%$, specificity to $92 \%$, and overall classification to $89 \%$. Finally, when segmentation data were added to the model, cGM $(\mathrm{p}<0.05)$ further improved sensitivity to $93 \%$, specificity to $95 \%$, and overall classification to $94 \%$. Finally, AD and MCI were classified with $74 \%$ sensitivity, $70 \%$ specificity, and an overall classification of $72 \%$ using the hippocampus $(\mathrm{p}<0.01)$ alone. Adding the ERC to the model, the ERC significantly contributed to the discrimination, and increased sensitivity to $81 \%$, specificity to $85 \%$, and overall classification to $83 \%$. Finally, when segmentation data were added to the model, no segmented structure contributed to the discrimination between $\mathrm{AD}$ and MCI.

\section{Discussion}

The major findings of this study were (1) the ERC and hippocampal volumes were significantly reduced in MCI compared with NC.

Table 4 Discrimination by volumes of entorhinal cortex and hippocampus and segmentation data

\begin{tabular}{|c|c|c|c|c|c|c|c|c|c|}
\hline & \multicolumn{3}{|c|}{$M C I$ and $N C$} & \multicolumn{3}{|c|}{$A D$ and $N C$} & \multicolumn{3}{|c|}{$A D$ and $M C I$} \\
\hline & Sensitivity & Specificity & Classn & Sensitivity & Specificity & Classn & Sensitivity & Specificity & Classn \\
\hline $\mathrm{HP}$ & $52 \%$ & $79 \%$ & $70 \%$ & $78 \%$ & $90 \%$ & $86 \%$ & $74 \%$ & $70 \%$ & $72 \%$ \\
\hline HP+ERC & $52 \%$ & $79 \%$ & $70 \%$ & $78 \%$ & $92 \%$ & $89 \%$ & $81 \%$ & $85 \%$ & $83 \%$ \\
\hline $\mathrm{HP}+\mathrm{SEG}$ & $59 \%$ & $82 \%$ & $77 \%$ & $93 \%$ & $95 \%$ & $94 \%$ & $81 \%$ & $85 \%$ & $83 \%$ \\
\hline
\end{tabular}

Logistic regression is used to calculate sensitivity, specificity, and classification (classn).

$\mathrm{ERC}=$ Entorhinal cortex; $\mathrm{HP}=$ hippocampus; $\mathrm{SEG}=$ segmentation; $\mathrm{NC}=$ normal cognition; $\mathrm{MCI}=$ mild cognitive impairment; $\mathrm{AD}=$ Alzheimer's disease. 
The magnitude of ERC atrophy was similar to that of hippocampal atrophy in MCI. (2) The ERC volume losses were greater than hippocampal volume losses in AD compared with NC. (3) There was significant volume loss in the ERC and hippocampus in $\mathrm{AD}$ compared with MCI. (4) There was significant correlation between the ERC and hippocampus in MCI and AD, not in NC. (5) Finally, the classifications between MCI versus NC, and $\mathrm{AD}$ versus $\mathrm{NC}$ were improved when segmentation data were added to the hippocampus. However, adding the ERC was only useful to improve the classification between $\mathrm{AD}$ and NC.

ERC AND HIPPOCAMPUS IN MCI COMPARED WITH NC

The first major finding of this study was that the ERC and hippocampal volume was significantly reduced in MCI compared with NC. Patients with MCI are generally considered to represent a non-demented group with a high likelihood of progressing to AD. Previous studies have shown that the ERC and hippocampus were significantly reduced in MCI compared with NC. ${ }^{1922} 2326$ However, the discriminatory power of the ERC reported before between MCI and NC was quite different. $\mathrm{Xu}$ et al reported that overall classification with the ERC between MCI and NC was less than $70 \%,{ }^{19}$ however, Killiany et al reported that overall classification between MCI and NC with the ERC was more than $85 \% .{ }^{26}$ Our study confirmed that the ERC and hippocampus were significantly reduced in MCI compared with NC. In addition, we also showed that reductions of the ERC and hippocampus were of similar magnitude and no trend of laterality of the ERC and hippocampal atrophy in MCI existed. However, there remains a considerable overlap in both ERC and hippocampal volume between MCI and NC. Furthermore, an overall classification of $70 \%$ between MCI and NC is moderate given that $50 \%$ classification can be achieved by chance. Both McNemar's test and logistic regression showed that the hippocampus was better than the ERC to distinguish MCI from NC and even adding the ERC to the hippocampus did not improve classification. This suggests that the ERC offers no advantage over the hippocampus in differentiating MCI from NC.

COMPARISON OF THE ERC AND HIPPOCAMPUS IN AD AND NC

The second major finding of this study was that there was significantly greater volume loss of the ERC than the hippocampus in $\mathrm{AD}$ compared with NC. Many brain structures, including the frontal lobe, parietal lobe, temporal lobe ${ }^{30-32}$ hippocampus, ERC, and amygdala have been measured using MRI in $\mathrm{AD}^{4-18}$ In general, the limbic structures of the hippocampus, ERC, and amygdala have shown the greatest changes. However, there has been considerable disagreement concerning the changes of the ERC and hippocampus in AD. ${ }^{41218} 19$ These differences probably resulted from different measured methods and different subjects. Our results show that ERC changes in $\mathrm{AD}$ were significantly larger than hippocampal changes. However, larger ERC changes may not be superior to hippocampal changes for the classification of $\mathrm{AD}$ from $\mathrm{NC}$, because the variability in measurement of the ERC is larger than that of the hippocampus. This is further substantiated by McNemar's tests, which showed no significant difference between the ERC and hippocampus for discriminating AD from NC. However, the classification between $\mathrm{AD}$ and $\mathrm{NC}$ was significantly improved after the ERC and hippocampus were combined, implying that the ERC is a useful marker to distinguish $\mathrm{AD}$ from $\mathrm{NC}$.

COMPARISON OF ERC AND HIPPOCAMPUS IN AD AND MCI

The third major finding of this study was that there was significant reduction of the ERC and hippocampus in $\mathrm{AD}$ when compared with MCI. Our study showed that there was a 30\% reduction of the ERC and $19 \%$ reduction of the hippocampus in $\mathrm{AD}$ compared with MCI. Both the ERC and the hippocampus could distinguish MCI from AD. This is consistent with previous studies. ${ }^{19}{ }^{25}$ Furthermore, the ERC had greater volume losses than the hippocampus in AD compared with MCI. Discrimination analysis also showed that the ERC had greater discrimination power than the hippocampus in separating AD and MCI. This was not consistent with the finding of $\mathrm{Xu}$ et al that the ERC and hippocampus had equivalent power to distinguish $\mathrm{AD}$ from $\mathrm{MCI} .{ }^{19}$

SEGMENTATION IN NC, MCI, AND AD

There was significant cGM loss and vCSF enlargement in both MCI and AD compared with NC. These findings were consistent with a previous study. ${ }^{15}$ The MRI findings indicate that patients with MCI had similar, but less severe, global cerebral changes (as measured by tissue segmentation) as well as focal cerebral changes (as determined by manual volume measurement of the ERC and hippocampus) than $\mathrm{AD}$, implying that the pathological process underlying MCI and $\mathrm{AD}$ are similar but with different severity.

CORRELATION OF THE ERC AND HIPPOCAMPUS IN NC, MCI, AND AD

The fourth major finding was that there were similar significant correlations between the ERC and hippocampus in MCI and AD, but not in NC. Necropsy studies of brains from patients with $\mathrm{AD}$ implied early pathology in the ERC with progression to the hippocampus. ${ }^{3}$ The conversion rate of MCI to $\mathrm{AD}$ has been reported to be $12 \%$ in 1 year and $19.5 \%$ in 2.7 years. ${ }^{27}$ The significant correlation of the ERC and hippocampus in MCI and AD is consistent with the view that $\mathrm{AD}$ pathology affects both these structures in parallel in MCI and AD. Recent reports suggested that hippocampal volume changes may help to predict MCI conversion to $\mathrm{AD},{ }^{24}{ }^{25}$ but we have no data on this matter in this cross sectional study. 
CLASSIFICATION OF GROUPS BY ERC,

HIPPOCAMPUS, AND SEGMENTATION

The fifth major finding was that the classifications between $\mathrm{MCI}$ versus $\mathrm{NC}$, and $\mathrm{AD}$ versus NC were improved after combination of hippocampal volumes with segmentation data, whereas the ERC only contributed to the classification between $\mathrm{AD}$ and NC. This suggests that combinations of different brain morphological changes assist classification between groups of NC, MCI, and $\mathrm{AD}$. However, $100 \%$ discrimination between the groups could not be achieved, as reported previously. ${ }^{34-36}$

This study had several limitations. Firstly, the patients with $\mathrm{AD}$ and those with MCI were not followed up to postmortem, so that a pathological diagnosis could not be made. Secondly, patients with MCI were not followed up longitudinally to test whether they ultimately developed AD. Finally, volume change may be not a specific marker of neuronal degeneration. Measurement of metabolic changes, especially the neuron specific amino acid $N$-acetyl aspartate using MR spectroscopy or spectroscopic imaging, ${ }^{11}{ }^{37-39}$ or functional changes such as perfusion or diffusion may further improve the diagnosis of MCI or AD. ${ }^{40-42}$

In conclusion, the ERC did not help the hippocampus to distinguish MCI from NC. However, the ERC was a better marker than the hippocampus in distinguishing $\mathrm{AD}$ from $\mathrm{MCI}$ and similar to the hippocampus in distinguishing $\mathrm{AD}$ from NC. Classifications between MCI and $\mathrm{NC}$, and $\mathrm{AD}$ and $\mathrm{NC}$ were improved after the ERC and hippocampus were combined with brain tissue segmentation data.

This work was supported in part by NIH grants AG10897, AG12435, the Alzheimer's Association, and Research Council for Health of the Academy of Finland. We gratefully acknowledge statistical assistance from Dr Peter Bachetti and segmentation assistance from Ms Mary Beth Kedzior.

1 Haroutunian V, Purohit DP, Perl DP, et al. Neurofibrillary tangles in non-demented elderly subjects and mild Alzheimer's disease. Arch Neurol 1999;56:713-18.

2 Haroutunian V, Perl DP, Purohit DP, et al. Regional distribution of neuritic plaques in the non-demented elderly and subjects with very mild Alzheimer's disease. Arch Neurol 1998;55:1185-91.

3 Braak H, Braak E, Bohl J. Staging of Alzheimer-related cortical destruction. Eur Neurol 1993;33:403-8.

4 Juottonen K, Laakso MP, Partanen K, et al. Comparative MR analysis of the entorhinal cortex and hippocampus in diagnosing Alzheimer disease. AfNR Am $\mathcal{f}$ Neuroradiol 1999;20:139-44

5 Juottonen K, Laakso MP, Insausti R, et al. Volumes of the entorhinal and perirhinal cortices in Alzheimer's disease. Neurobiol Aging 1998;19:15-22.

6 Krasuski JS, Alexander GE, Horwitz B, et al. Volumes of medial temporal lobe structure in patients with Alzheimer's disease and mild cognitive impairment (and in healthy disease and mild cognitive impairment

7 Kaye JA, Swihart T, Howieson D, et al. Volume loss of the hippocampus and temporal lobe in the healthy elderly persons destined to develop dementia. Neurology 1997;48 1297-304

8 Jack CR Jr, Petersen RC O'Brien PC, et al. MR-based hippocampal volumetry in the diagnosis of Alzheimer's disease. Neurology 1992;42:183-8.

9 Jack CR Jr, Petersen RC, Xu Y, et al. Rate of medial temporal lobe atrophy in typical aging and Alzheimer's disease. Neurology 1998;51:993-9.

10 Laakso MP, Soininen H, Partanen K, et al. Volumes of hippocampus, amygdala and frontal lobes in the MRI-based diagnosis of early Alzheimer's disease: correlation with memory functions. $\mathcal{F}$ Neural Transm Park Dis Dement Sect 1995;9:73-86.

11 Schuff N, Amend D, Ezekiel F, et al. Change of hippocampal N-acetyl aspartate and volume in Alzheimer's disease. Neurology 1997;49:1513-21.

12 Frisoni GB, Laakso MP, Beltramello A, et al. Hippocampal and entorhinal cortex atrophy in frontotemporal dementia and Alzheimer's disease. Neurology 1999;52:91-100.
13 Jack CR Jr, Petersen RC, Xu YC, et al. Medical temporal atrophy on MRI in normal aging and very mild Alzheimer's disease. Neurology 1997;49:786-94.

14 de Leon MJ, Convit A, De Santi S, et al. Contribution of structural neuroimaging to the early diagnosis of Alzheimer disease. Int Psychogeriatr 1997;9:183-90.

15 Tanabe JL, Amend D, Schuff N, et al. Tissue segmentation of the brain in Alzheimer's disease. AfNR Am f Neuroradiol 1997; 18:115-23.

16 Barber R, Scheltens P, Gholkar A, et al. White matter lesions on magnetic resonance imaging in dementia with Lewy bodies, Alzheimer's disease, vascular dementia, and normal aging. F Neurol Neurosurg Psychiatry 1999;67:66-72.

17 Kidron D, Black SE, Stanchev P, et al. Quantitative MR volumetry in Alzheimer's disease. Topographic markers and the effects of sex and education. Neurology 1997;49:150412 .

18 Bobinski M, de Leon MJ, Convit A, et al. MRI of entorhinal cortex in mild Alzheimer's disease. Lancet 1999;353:38-40.

$19 \mathrm{Xu}$ Y, Jack CR Jr, O'Brien PC, et al. Usefulness of MRI measures of entorhinal cortex versus hippocampus in AD. Neurology, 2000;54:1760-7.

20 Fox NC, Warrington EK, Stevens JM, et al. Atrophy of the hippocampal formation in early familial Alzheimer's disease. A longitudinal MRI study of at-risk members of a family with an amyloid precursor protein 717Val-Gly mutation. Ann NY Acad Sci 1996;777:226-32

21 Reiman EM, Uecker A, Caselli RJ, et al. Hippocampal volumes in cognitively normal persons at genetic risk for Alzheimer's disease. Ann Neurol 1998;44:288-91.

22 Convit A, de Leon MJ, Tarshish C, et al. Hippocampal volume losses in minimally impaired elderly. Lancet 1995;345: 266.

23 Convit A, de Leon MJ, Tarshish C, et al. Specific hippocampal volume reduction in individuals at risk for Alzheimer's disease. Neurobiol Aging 1996;18:131-8.

24 Jack CR Jr, Petersen RC, Xu YC, et al. Prediction of AD with MRI-based hippocampal volume in mild cognitive impairment. Neurology 1999;52:1397-403.

25 Visser PJ, Scheltens P, Verhey FR, et al. Medical temporal obe atrophy and memory dysfunction as predictors for dementia in subjects with mild cognitive impairment. $\mathcal{f}$ Neurol 1999;246:477-85.

26 Killiany RJ, Gomez-Isla T, Moss M, et al. Use of structural magnetic resonance imaging to predict who will get Alzheimer's disease. Ann Neurol 2000;47:430-9.

27 Petersen RC, Smith GE, Waring SC, et al. Mild cognitive impairment: clinical characterization and outcome. Arch Neurol 1999;56:303-8.

28 Insausti $\mathrm{R}$, Juottonen $\mathrm{K}$, Soininen $\mathrm{H}$, et al. MR volumetric analysis of the human entorhinal, perirhinal, and temporopolar cortices. AfNR Am f Neuroradiol 1998;19:659-71.

29 Watson C, Andermann F, Gloor P, et al. Anatomic basis of amygdaloid and hippocampal volume measurement by magnetic resonance imaging. Neurology 1992;42:1743-50.

30 Pantel J, Schröder J, Schad LR, et al. Quantitative magnetic resonance imaging and neuropsychological function in dementia of the Alzheimer type. Psychol Med 1997;27:221-

31 Kitagaki H, Mori E, Yamaji S, et al. Frontotemporal dementia and Alzheimer's disease: evaluation of cortical atrophy with automatic hemispheric surface display generated with MR imaging. Radiology 1998;208:431-9.

32 Geroldi C, Pihlajamäki M, Laakso MP, et al. APOE- ${ }^{\circ} 4$ is associated with less frontal and more medical temporal lobe atrophy in AD. Neurology 1999;53:1825-32.

33 Wolf $\mathrm{H}$, Grunwald $\mathrm{M}$, Ecke GM, et al. The prognosis of mild cognitive impairment in the elderly. $\mathcal{F}$ Neural Transm (Suppl) 1998;54:31-50.

34 Pearlson GD, Harris GJ, Powers RE, et al. Quantitative changes in mesial temporal volume, regions cerebral flow, and cognition in Alzheimer's disease. Arch Gen Psychiatry 1992;49:402-8.

35 Lehéricy S, Baulac M, Chiras J, et al. Amygdalohippocampal MR volume measurements in early stages of Alzheimer disease. AFNR Am 7 Neuroradiol 1994; 15:927-37.

36 Kesslak JP, Nalcioglu O, Cotman CW. Quantification of magnetic resonance scans for hippocampal and parahippocampal atrophy in Alzheimer's disease. Neurology 1991; 41:51-4.

37 Schuff N, Amend DL, Meyerhoff DJ, et al. Alzheimer disease: quantitative $\mathrm{H}-1 \mathrm{MR}$ spectroscopic imaging of frontoparietal brain. Radiology 1998;207:91-102.

38 MacKay S, Meyerhoff DJ, Constans JM, et al. Regional gray and white matter metabolite differences in subjects with $\mathrm{AD}$, with subcortical ischemic vascular dementia, and elderly controls with $1 \mathrm{H}$ magnetic resonance spectroscopic imaging. Arch Neurol 1996;53:167-74.

39 MacKay S, Ezekiel F, Di Sclafani V, et al. Alzheimer disease and subcortical ischemic vascular dementia: evaluation by combining MR imaging segmentation and $\mathrm{H}-1$ MR spectroscopic imaging. Radiology 1996;198:537-45.

40 Sandson TA, O'Connor M, Sperling RA, et al. Non-invasive perfusion MRI in Alzheimer's disease: a preliminary report. Neurology 1996;47:1339-42.

41 Hanyu $\mathrm{H}$, Sakurai H, Iwamoto T, et al. Diffusion-weighted MR imaging of the hippocampus and temporal white matter in Alzheimer's disease. 7 Neurol Sci 1998;156:195-200.

42 Sandson TA, Felician O, Edelman RR, et al. Diffusionweighted magnetic resonance imaging in Alzheimer's disease. Dement Geriatr Cogn Disord 1999;10:166-71. 